\title{
Changing the Landscape of Medical Oncology Training at the National University Hospital in the Philippines during the Coronavirus Disease 2019 (COVID-19) Pandemic
}

\author{
Alfredo Chua Jr. ${ }^{1}$ (I) Marvin Jonne Mendoza ${ }^{1} \cdot$ Mark Ando $^{1} \cdot$ Cyril Jonas Planilla ${ }^{1} \cdot$ Gracieux Fernando ${ }^{1}$. \\ Heinrik Martin Jude Strebel ${ }^{1}$. Jorge Ignacio ${ }^{1}$
}

(C) American Association for Cancer Education 2020

\begin{abstract}
Serving as one of the few training institutions of medical oncology in the Philippines, the University of the Philippines-Philippine General Hospital was faced with challenges brought by the coronavirus 2019 disease (COVID-19) pandemic. With the dismantling of routines and practices in the hospital, training activities such as daily rounds, conferences, and examinations were temporarily put on hold. Recognizing that the strength of any clinical training program is its wealth of patients, the immediate resumption of patient services, albeit limited at first, had been instrumental in ensuring the continuation of training in our institution. Opportunistic teaching-learning strategies between the faculty and fellows were devised. Innovative approaches to learning such as the use of online meeting platforms for division conferences, webinars, examinations, and other learning activities were initiated. Emphasis was given on the important considerations in the management of cancer patients during the COVID-19 pandemic. The emotional and psychological well-being of the faculty and fellows during this crisis were considered and a mental health assessment was conducted prior to the resumption of training activities.
\end{abstract}

\section{Training during the Pre-pandemic Setting}

The University of the Philippines-Philippine General Hospital, Division of Medical Oncology serves as one of the few training institutions in the Philippines for internists pursuing further subspecialty training in medical oncology. The division has 12 to 14 fellows for its 2-year training program. They alternately rotate in different in-patient services with daily outpatient clinics. They also attend multidisciplinary team meetings to discuss cases and develop treatment plans. Didactics include bimonthly journal reports and examinations which serve as formative evaluations. In all these training activities, they are continuously guided by the faculty. Furthermore, the Philippine Society of Medical Oncology

Alfredo Chua, Jr.

avchua@up.edu.ph

1 Division of Medical Oncology, Department of Medicine, University of the Philippines - Philippine General Hospital, Taft Avenue, Manila, Philippines
(PSMO) designates topics to each training institution for monthly round-table discussions tackling interesting cases with dilemmas in management. Attendance in webinars, grand rounds, and other continuing medical education (CME) activities are likewise encouraged. In addition, they also produce research outputs intended for presentation and publication.

The COVID-19 pandemic has dismantled routines and practices in hospitals worldwide. The Philippine General Hospital has been delegated as a COVID-19 referral center, with subsequent conversion of various areas for COVID-19 patients. There was cessation of non-essential services, including elective admissions for oncologic treatment. All efforts, manpower, and resources were focused in addressing the COVID-19 crisis [1]. Fellows and consultants were called to augment the workforce and serve as front liners. With these constraints, most training activities were temporarily put on hold.

In this paper, we discuss the challenges faced by the Division of Medical Oncology at the national university hospital of the Philippines in terms of subspecialty training and how it has adapted to these difficult predicaments. 


\section{Continuity in the Rupture}

One of the challenges in training during this pandemic was ensuring the safe entry of newly accepted fellows into the training program. Planning a smooth transition as they familiarize themselves with the day-to-day activities of an oncology fellow-in-training was difficult given that the entire healthcare system was overhauled. Because of the enhanced community quarantine (ECQ), new fellows coming from the provinces had difficulty in domestic travel with limited options in accommodation within the capital.

The learning environment has not been ideal because of the limited encounters with patients. The Cancer Institute was temporarily closed, and upon resumption of services last April 1, 2020 , there was a significant reduction in the number of patients seen daily from the usual 150 to 200 patients, to a maximum of 25 patients only. Clinic-based learning became a challenge. Daily rounds were fewer because admissions were limited to COVID-19 patients. This imposed pressure to the new fellows who needed to quickly adjust to the new responsibilities while rotating at the COVID-19 areas. Training activities such as monthly round-table discussions, journal reporting, and examinations have also been affected. Conferences such as the PSMO midyear convention were also postponed.

All healthcare professionals were subjected to a plethora of thoughts and emotions - more than the usual they need to face on a normal day. Feelings of anxiety, fear, uncertainty, isolation, and burnout were common. Others may feel vindicated that it is their duty to serve the people in this pandemic. Others may have reservations given the high rate of infection among healthcare workers [1].

\section{Changing the Landscape and Adapting to the "New Normal"}

A change in the learning paradigm must be sought to adapt to the new architecture the pandemic has brought. At the core of any clinical training program is the wealth of patients walking through its doors, carrying different clinical scenarios, and presenting as opportunities for learning. With the ECQ and the temporary closure of the Cancer Institute, the medical oncology training program was essentially put on hold starting the second week of March. During this time, planning for alternative strategies through weekly online meetings was done. The resumption of patient services, albeit limited at first, has been instrumental in ensuring the continuation of training. Prioritizing the safety of both patients and healthcare providers, continuation of cancer care amidst the COVID-19 pandemic has been the overall goal [2-4]. Strict safety precautions like patient prioritization, screening protocols, and social distancing measures were observed allowing the slow but safe resumption of services. Patients not needing urgent care who can be managed remotely were attended to through teleconsultations [5]. Eventually, multidisciplinary clinics resumed, and conferences were scheduled via an online meeting platform.

There was a need to devise opportunistic teaching-learning strategies between the faculty and fellows. The aphorism, "every patient is a learning opportunity," has never been truer. Taking advantage of the limited number of patients, bedside rounds were continued if possible. Several consultants were assigned per week to go to the clinics and wards to teach. A buddy system between the faculty and fellows was started,
Table 1 Challenges and adaptations done for the medical oncology training at the national university hospital in the Philippines

\begin{tabular}{|c|c|}
\hline Challenges & Adaptations \\
\hline $\begin{array}{l}\text { - PGH delegated as COVID-19 referral center and } \\
\text { temporary closure of the Cancer Institute with cessation } \\
\text { of non-essential services such as chemotherapy }\end{array}$ & $\begin{array}{l}\text { - Weekly division meetings for strategic planning } \\
\text { - Gradual resumption of patient services with strict } \\
\text { safety precautions and screening protocols }\end{array}$ \\
\hline $\begin{array}{l}\text { - Crisis coincided with start of new fellows making } \\
\text { adjustment to new roles difficult }\end{array}$ & $\begin{array}{l}\text { - Formation of buddy system between faculty, } \\
\text { senior and junior fellows } \\
\text { - Creation of a practical guide on basic } \\
\text { chemotherapy }\end{array}$ \\
\hline $\begin{array}{l}\text { - Significant reduction in the number of patients seen } \\
\text { limiting clinic-based learning opportunities }\end{array}$ & $\begin{array}{l}\text { - Adaptation of opportunistic teaching-learning } \\
\text { strategies } \\
\text { - Provision of reading assignments and materials } \\
\text { - Initiation of monthly case presentations and } \\
\text { discussions }\end{array}$ \\
\hline $\begin{array}{l}\text { - Postponement of conferences, monthly round-table } \\
\text { discussions, journal reports, and examinations }\end{array}$ & $\begin{array}{l}\text { - Utilization of online platforms for CME } \\
\text { activities and formative evaluations }\end{array}$ \\
\hline $\begin{array}{l}\text { - Possible psychological effects of the pandemic on faculty } \\
\text { and fellows }\end{array}$ & $\begin{array}{l}\text { - Evaluation of mental health and psychosocial } \\
\text { processing to assess readiness for training }\end{array}$ \\
\hline $\begin{array}{l}\text { - Temporary interruption of ongoing clinical trials and } \\
\text { research projects }\end{array}$ & $\begin{array}{l}\text { - Prioritization of topics relating to cancer care } \\
\text { during the COVID-19 pandemic }\end{array}$ \\
\hline
\end{tabular}


with the consultant and senior fellow guiding the junior fellow during the first weeks of training. A practical guide on the basic principles of oncology care and chemotherapy was also provided.

The emotional and psychological well-being of the faculty and fellows was also factored into planning $[4,6]$. Another important step was assessing the mental health of the faculty and fellows before resuming training activities. In cooperation with the Department of Psychiatry and Behavioral Medicine, a program was created, and separate virtual meetings with the first- and second-year fellows, and consultants were conducted. These served as a processing of their psychosocial reactions to the pandemic and an assessment of their readiness to proceed and transition back to the training activities. Coping strategies and potential problems that could impede with their readiness were also explored.

The radical limitation imposed by this crisis paved the way for other innovative approaches to learning. During the time of temporary closure, suggested reading assignments were given. Attendance in PSMO-led webinars and online activities were encouraged providing avenues for academic discussions and learning. The bimonthly journal reports were resumed in May 2020 via an online platform. Instead of giving an actual written test, examinations were sent online. To further reinforce learning and compensate for the limited number of patients, monthly case presentations and discussions were started. Cases were presented virtually and important points in management were discussed. An online platform allowed fellows who were relegated to other areas or in self-isolation or quarantine, to still learn whether by attending live or by accessing the recorded sessions [5].

During discussions, emphasis was given on the important considerations in the management of cancer patients during the COVID-19 pandemic. Cancer patients are more susceptible to infections brought about by their disease and the immunosuppressive effects of treatment [3]. Balancing the need for chemotherapy and the risk of contracting the infection is complicated [6]. Whether to initiate, continue, or delay cancer treatment must be carefully planned. Guidance from the faculty in making these clinical decisions is important. Multidisciplinary team meetings were also helpful in making these decisions.

Aside from the temporary interruption of ongoing clinical trials, the pandemic presented opportunities for research. Topics relating to cancer care during the pandemic were pursued. These include describing the profile of cancer patients with COVID-19, exploring the psychological impact to cancer patients, and looking at the oncologic outcomes of patients whose treatments were affected.

Table 1 summarizes the challenges during the COVID-19 pandemic vis-à-vis the adaptations done to address these challenges.

\section{Conclusions}

The COVID-19 pandemic has brought changes of great impact to our society. The training of medical oncologists around the world is no exception. The landscape of medical oncology training worldwide has already changed. The combination of effective traditional and innovative teaching-learning strategies is expected to become the new paradigm. Institutions should adapt to safeguard the training of soon-to-be medical oncologists. With good leadership, strategic planning, and collaboration, challenges can become opportunities despite the uncertainty of these trying times. The landscape may have changed but the goal of maintaining the quality of training will always remain.

Code Availability (Software Application or Custom Code) Not applicable.

Authors' Contributions Alfredo Chua Jr. and Marvin Jonne Mendoza conceptualized the topic. The first draft of the manuscript was written by Alfredo Chua Jr., Marvin Jonne Mendoza, Mark Ando, and Cyril Jonas Planilla. Gracieux Fernando, Heinrik Martin Jude Strebel, and Jorge Ignacio commented on previous versions of the manuscript. All authors read and approved the final manuscript.

Data Availability Not applicable.

\section{Compliance with Ethical Standards}

Conflicts of Interest The authors declare that they have no conflict of interest.

\section{References}

1. Ting F, Fernando GY (2020) Double trouble: challenges of cancer care in the Philippines during the COVID-19 pandemic. EJMO 4(2): 135-136

2. Francesco C, Pettke A, Michele B et al (2020) Managing COVID-19 in the oncology clinic and avoiding the distraction effect. Ann Oncol. https://doi.org/10.1016/j.annonc.2020.03.286

3. Lambertini M, Toss A, Passaro A, Criscitiello C, Cremolini C, Cardone C, Loupakis F, Viscardi G, Meattini I, Dieci MV, Ferrara R, Giusti R, Maio MD (2020) Cancer care during the spread of coronavirus disease 2019 (COVID-19) in Italy: young oncologists' perspective. ESMO Open 5:e00759. https://doi.org/10.1136/ esmoopen-2020-000759

4. Ueda M, Martins R, Hendrie PC, McDonnell T, Crews JR, Wong TL, McCreery B, Jagels B, Crane A, Byrd DR, Pergam SA, Davidson NE, Liu C, Stewart FM (2020) Managing cancer care during the COVID-19 pandemic: agility and collaboration toward a common goal. J Natl Compr Cancer Netw 18:366-369

5. DeFilippis EM, Stefanescu Schmidt AC, Reza N (2020) Adapting the educational environment for cardiovascular fellows-in-training during the COVID-19 pandemic. J Am Coll Cardiol 75:26302634. https://doi.org/10.1016/j.jacc.2020.04.013

6. Burki TK (2020) Cancer guidelines during the COVID-19 pandemic. Lancet Oncol 21(5):629-630

Publisher's Note Springer Nature remains neutral with regard to jurisdictional claims in published maps and institutional affiliations. 